\title{
Computer-Assisted Three-Dimensional Reconstruction of the Vestibular End-Organs of the Gerbil Using the Serial Paraffin Sections
}

\author{
Noriko Kawamura, Akiko Seto-Ohshima and Muneyuki Ito \\ Institute for Developmental Research, Aichi Human Service Center, Kasugai 480-03
}

Received for publication November 11, 1996 and in revised form February 7, 1997

\begin{abstract}
To obtain spatial information regarding gerbil vestibular end-organs and surrounding components, serial paraffin sections of the decalcified heads, including inner ear, were prepared. Photographs of the sections were traced onto OHP papers, on which two reference points were plotted for the orientation. Sets comprised of a photograph and an OHP sheet with two reference points were digitized using a scanner and the computer program, "Photoshop". The figures

were cropped to the same size using two reference points and after the color density of the objects was increased, they were superimposed using the computer program, "VoxelView" for three-dimensional reconstruction. This was an effective method of clarifying the spatial relationship of the components included. Using the results obtained, precise experiments for electrical vestibular stimulation were successfully performed.
\end{abstract}

Key words: Three-dimensional structure, Vestibular end-organs, Gerbil

\section{Introduction}

Recent developments in the field of computer programming now make it possible to study and understand the complicated structures found in living organisms and have contributed to both basic and applied sciences such as medicine. In the present study, we used one such program, "VoxelView", now available for the basic laboratory without requiring large dedicated work-station, as used in [1], to reconstruct the three-dimensional (3-D) configuration of the vestibular end-organs and surrounding structures in the Mongolian gerbil (Meriones unguiculatus). The gerbil is a genetic animal model of epilepsy, which we use to study the electrophysiological responses elicited in the cerebral cortex by various experimental stimuli to elucidate the induction mechanism of epileptic electrical discharge in the cerebral cortex. It was demonstrated that computer-assisted images can provide the precise spacial information required for specific electrical stimulation of the vestibular system.

Correspondence to: Dr. Akiko Seto-Ohshima, Institute for Developmental Research, Aichi Human Service Center, 713-8 Kamiyacho, Kasugai, Aichi 480-03, Japan.

\section{Materials and Methods}

The gerbils used were from a colony of a seizuresusceptible strain MGS/Idr [7]. They were anesthetized on postnatal day 60 using sodium pentobarbital $(75 \mu \mathrm{g} / \mathrm{g}$ body weight), then perfused transcardially with saline, followed by $4 \%$ paraformaldehyde in $0.1 \mathrm{M}$ phosphate buffer (pH 7.4). The skin and most of the head muscle were removed and the heads were fixed for 2 more days in the same fixative at $4^{\circ} \mathrm{C}$, treated for $1 \mathrm{hr}$ with $70 \%$ alcohol, then immersed in $10 \%$ formic acid at $37^{\circ} \mathrm{C}$. Some specimens were treated with $10 \%$ EDTA ( $\mathrm{pH} \mathrm{7.4)} \mathrm{instead}$ of formic acid. When bones of these heads were soft enough to cut easily with a razor, a frontal cut was made at the level of the superior colliculus and the blocks were processed for paraffin embedding. Five consecutive frontal sections ( $7 \mu \mathrm{m}$ thick) from each specimen were mounted on a glass slide. The sections on the glass slides of odd number were stained using the Klüver-Barrera method. Some of them were used for 3-D reconstruction. With the remaining sections, those on every tenth slide were stained with heamatoxylin and eosin, and a few sections were treated with Bodian stain for neuronal constituents or Gomori's one stage trichrome stain for collagen and 
myeline [6].

For computer-assisted reconstruction of the 3-D structure, 26 frontal sections taken at $70 \mu \mathrm{m}$ intervals starting at the point of first appearance of the anterior part of vestibular ganglion (anterior) and ending at the end of the ampullary crista of the posterior semicircular duct (posterior), were photographed. A sheet of OHP paper was then placed on each print and the outline of the print itself and that of several arbitrarily chosen structures, such as the skull, the brain proper and/or structures that showed the orientation to maintain the continuity of the materials on the z-axis, were drawn. The sheets of OHP paper were then stacked in order, maintaining the z-axis. On each sheet, two reference points were plotted through the series. A second series of OHP paper was laid over the first series and the position of the two reference points and the outline of the print were traced as in the first series but the outline of the structures were omitted. The OHP sheets from this second series were then matched to the corresponding print by the traced outline; each set was then digitized using a scanner (ScanJet $4 \mathrm{c} / \mathrm{T}$, Hewlett-Packard Company, CO, U.S.A.) and the computer program

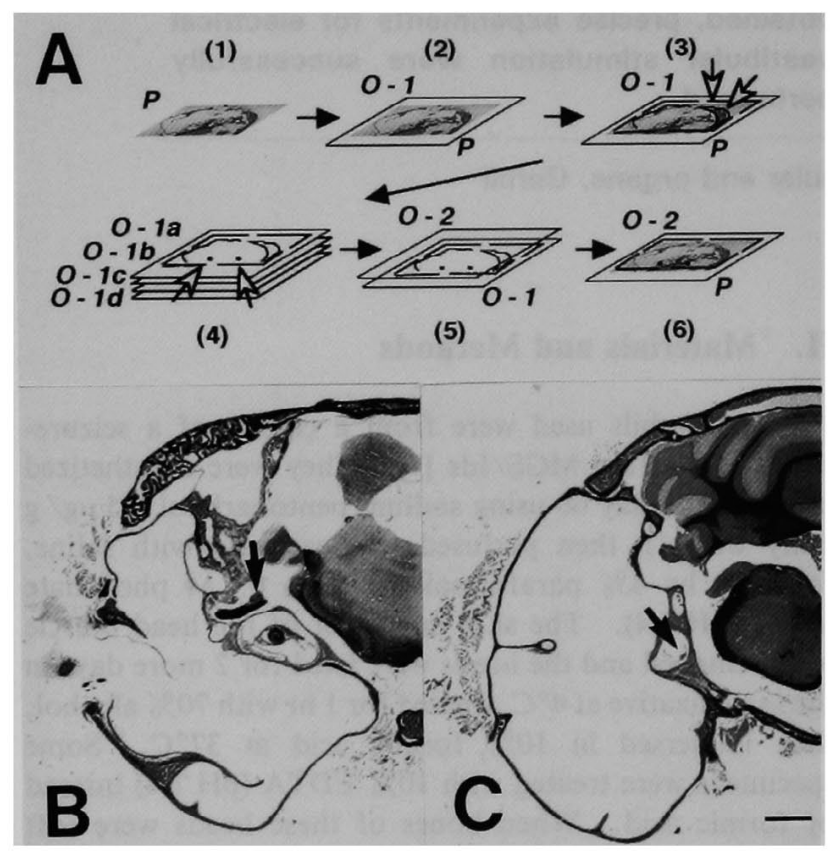

Fig. 1. Schematic representation of the processes involved in aligning the figures and the first and the last figures used for 3-D reconstruction. (A) The process for aligning the figures to be superimposed using "VoxelView" is shown schematically. The white arrows indicate the outline of the print (in (3), left), that of several structures (in (3), right) and two reference points (in (4)) on the OHP paper, respectively. $P$ : print, $O-1 a-O-1 d$ : individual OHP papers (first series), O-2: OHP paper (second series). For details, see text. The first figure (B) with the first appearance of the vestibular ganglion (arrow) in contact with the facial nerve bundle and the last figure $(\mathrm{C})$ in which the ampullary crista of the posterior semicircular duct (arrow) ended are shown. Bar $=1 \mathrm{~mm}$.
"Photoshop" (Adobe System Inc., CA, U.S.A.) and cropped to precisely the same size using the two reference points. The computer used was Power Macintosh 8100/80AV (Apple Computer, Inc., CA, U.S.A.). These processes are shown schematically in Fig. 1. The color density of the objects described below was increased by hand to distinguish these objects from each other and from the surrounding structures after reconstruction; the vestibular ganglion and vestibular nerve (vestibular portion of the VIIIth nerve), cochlear nerve (part of cochlear portion of the VIIIth nerve), facial nerve, membranous semicircular ducts with their ampullary crista, macula of utricle and macula of saccule. Then, the figures were superimposed using the computer program "VoxelView" (Vital Images, Inc., IO, U.S.A.), which allows 3-D reconstruction of superimposed figures.

In the present case, a $17 \mu \mathrm{m}$ length in the original paraffin section corresponded to 1 pixel in the computerized figure. The exact distance from the top surface of a paraffin section to that of the next one was calculated to be $70 \mu \mathrm{m}$, which corresponded to 4 pixels. In this program, a single computerized figure occupied a thickness of 1 pixel in z-axis instead of the exact thickness of a paraffin section (7 $\mu \mathrm{m}, 0.4$ pixels). Therefore 3 further figures (total thickness, 3 pixels), which were generated automatically by the computer to maintain the continuity of the figures, were inserted between adjacent figures.

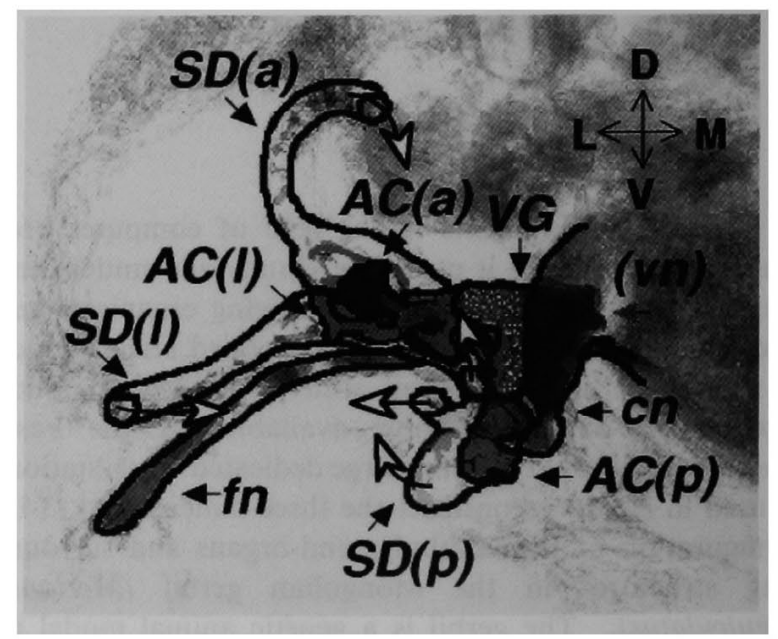

Fig. 2. Schematic illustration of a reconstructed figure. Schematic illustration of overall view of the reconstructed image of the vestibular end-organs and surrounding structures in the left semihemisphare was drawn. This figure corresponds to that observed in the posterior - anterior direction, allowing visualization of the ampullary crista of the posterior semicircular duct, normally hidden by the cochlear nerve. The original figure is (A) in Fig. 3. The direction of membranous semicircular ducts is shown with white arrows. $A C(a, l, p)$ : ampurally crista (anterior, lateral, posterior), $c n$ : cochlear nerve, $f n$ : facial nerve, $S D(a, l$, p): membranous semicircular duct (anterior, lateral, posterior), VG: vestibular ganglion, $v n$ : vestibular nerve. $\mathrm{D}$ : dorsal, $\mathrm{V}$ : ventral, L: lateral, M: medial. 


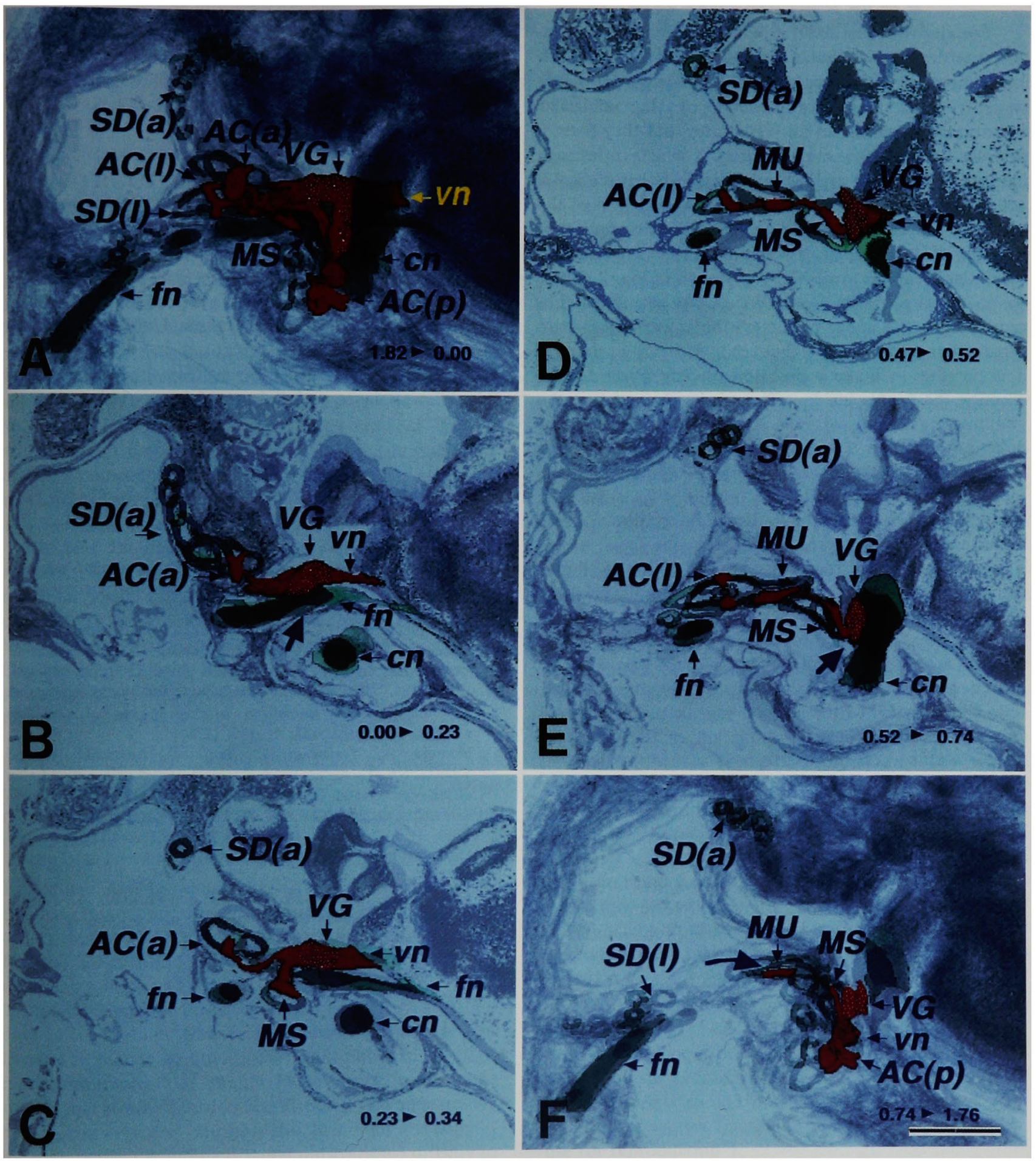

Fig. 3. Computer-assisted 3-D image of the vestibular end-organs of the gerbil. (A) The original figure of overall view of the reconstructed image. Vestibular nerve, vestibular ganglion, maculla of utricle, maculla of saccule and ampullary crista were colored red. Part of the vestibular nerve hidden by the cochlear nerve is shadowed. On the assumption that the thickness of a paraffin section is $7 \mu \mathrm{m}$, the entire thickness was calculated as $1.82 \mathrm{~mm}$ with the top being designated as $0 \mathrm{~mm}$. (B-F) Images seen in the anterior - posterior direction. (B) $0.00 \mathrm{~mm}-0.23 \mathrm{~mm}$ level. $($ (C) $0.23 \mathrm{~mm}-0.34 \mathrm{~mm}$ level. (D) $0.47 \mathrm{~mm}-0.52 \mathrm{~mm}$ level. (E) $0.52-0.74 \mathrm{~mm}$ level. (F) $0.74-1.76 \mathrm{~mm}$ level. The large arrows in (B) and (E) indicate the point where close contact of the vestibular ganglion and the facial (B) or the cochlear (E) nerve bundles was observed. The large arrow in (F) indicates the maculla of utricle to which the wire electrodes were directed for electrical stimulation. MS: maculla of saccule, $M U$ : maculla of utricle. Other abbreviations are same as in Fig. 2 . Bar $=1 \mathrm{~mm}$. 


\section{Results and Discussion}

For electrical vestibular stimulation, spatial information regarding the vestibular end-organs and the surrounding components is required to avoid stimulating other systems. The major constituents of the vestibular end-organs are similar in all vertebrates, but they form a complex structure, the configuration of which varies with the species [3]. The gerbil has large thin-boned bulla [4] and is used in experiments involving manipulation of the vestibular labyrinth [2]; however, no precise description of the spacial location of the major constituents of the vestibular system is available. As shown in Fig. 2 and 3, the computer program, "VoxelView", was able to assist in generating a 3-D image of the vestibular end-organs and surrounding components of the gerbil. Using this program, it is possible to make a partition on any plane and to examine the profile inside. The results showed that the vestibular ganglion extends in an anterior-posterior direction and that, at the anterior part of the vestibular ganglion, the vestibular nerve is in close contact with the facial nerve bundle. Therefore, this area was considered unsuitable for electrical stimulation of the vestibular nerve because of the high risk of stimulating the facial nerve. The same is true for the posterior part of the vestibular ganglion which carries the risk of stimulating the cochlear nerve bundle.

There was no detectable difference due to gender and the EDTA-treated material used to avoid the low pH treatment demonstrated identical findings. Using these results, following removal of the periotic capsule in anesthetized animals, specific electrical stimulation of the vestibular system was successfully performed via wire electrodes inserted into a cut made in the lateral semicircular duct towards the maculla of utricle as shown in Fig. 3F. Recently, Nomura et al. applied the "VoxelView" to the 3-D analysis of material on the stage of a con-focal microscope with which sections having a constant z-axis were prepared automatically [5]. For the use with a confocal microscope, choices regarding section thickness and methods for detecting objects (usually fluorescent staining) are limited. Our study shows that if sections are prepared carefully and sections are carefully aligned, "VoxelView" is a useful tool for reconstructing 3-D structures from an intermittent series of consecutive paraffin sections or from similar sections prepared in a basic laboratory by other methods.

\section{Acknowledgments}

The authors are grateful to Image and Measurement, Inc (Tokyo, Japan) for help with the 3-D reconstruction and acknowledge fruitful discussion and encouragement from Drs. N. Takeda, S. Horii (Osaka University), S. Saga, R. Kato-Semba (Institute for Developmental Research), H. Maeda (Aichi-Gakuin University) and A. Mizutani. This study was supported in part by a Grantin-Aide for Scientific Research from the Ministry of Education, Science and Culture of Japan (No. 05680748, 07640917).

\section{References}

1. Henick, D. H.: Three-dimensional analysis of murine laryngeal development. Ann. Otol. Rhinol. Laryngol. 102; 3-24, 1993.

2. Kaufman, G. D. and Perachio, A. A.: Translabyrinth electrical stimulation for the induction of immediately-early genes in the gerbil brainstem. Brain Res. 646; 345-350, 1994.

3. Kuhlenbeck, H.: IX. Medulla Oblongata (and Pons). Spinal Cord and Deuterencephalon. The Central Nervous System of Vertebrates, Vol. IV., S. Karger AG., Basel, 1975, pp. 288-623.

4. Lay, D. M.: The anatomy, physiology, functional significance and evolution of specialized hearing organs of gerbilline rodents. J. Morph. 138; 41-120, 1972.

5. Nomura, A., Yamazaki, Y., Tsuji, T., Kawasaki, Y. and Tanaka, S.: Three-dimensional labeling program for elucidation of the geometric properties of biological particles in three-dimensional space. Exp. Cell Res. 227; 256-263, 1996.

6. Ralis, H. M., Beesley, R. A. and Ralis, Z. A.: Techniques in Neurohistology, Butterworths \& Co. (Publishers) Ltd., London, 1973.

7. Seto-Ohshima, A., Ito, M., Kudo, T. and Mizutani, A.: Intrinsic and drug-induced seizures of adult and developing gerbils. Acta Neurol. Scand. 85; 311-317, 1992. 